\title{
ENDOMORPHISMS OF EXACT SEQUENCES ${ }^{1}$
}

BY IRWIN S. PRESSMAN

Communicated by I. N. Herstein, September 3, 1970

Let $E$ and $F$ denote the following exact sequences

$$
E: 0 \rightarrow A \stackrel{f}{\rightarrow} B \stackrel{g}{\rightarrow} C \rightarrow 0 \quad F: 0 \rightarrow A \stackrel{f}{\rightarrow} B \stackrel{h g}{\rightarrow} D \stackrel{j}{\rightarrow} E \rightarrow 0
$$

where $h g$ represents the canonical factorization of the middle morphism of $F$ into an epimorphism $g$ followed by a monomorphism $h$. We shall take the term "endomorphism" of $E$ or $F$ to mean a commutative diagram of the form

$$
\begin{aligned}
& 0 \rightarrow A \stackrel{f}{\rightarrow} B \stackrel{g}{\rightarrow} C \rightarrow 0 \quad 0 \rightarrow A \stackrel{f}{\rightarrow} B \stackrel{h g}{\rightarrow} D \stackrel{j}{\rightarrow} E \rightarrow 0
\end{aligned}
$$

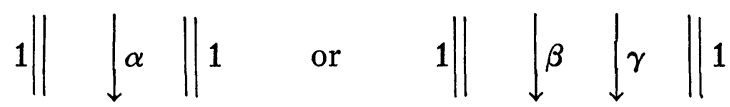

$$
\begin{aligned}
& 0 \rightarrow A \stackrel{f}{\rightarrow} B \stackrel{g}{\rightarrow} C \rightarrow 0 \quad 0 \rightarrow A \stackrel{f}{\rightarrow} B \stackrel{h g}{\rightarrow} D \stackrel{j}{\rightarrow} E \rightarrow 0 .
\end{aligned}
$$

We shall compute the "endomorphism groups" of $E$ and $F$ and prove that $\operatorname{Aut}(E)=\operatorname{End}(E) \cong \operatorname{Hom}(C, A)$ and that $\operatorname{End}(F) \cong \operatorname{Hom}(h, g)$ where the second Hom is a functor on a category of morphisms with range the category of semigroups.

1. Notation. Let $R$ denote a fixed ring with unit and $\Re$ the category of left $R$-modules. Let $\mathcal{E}$ denote the category of all short-exact sequences $E$, which begin with $A$ and end with $C$, and whose morphisms are all triples $(1, \theta, 1)=\theta^{\#}$ which induce commutative diagrams

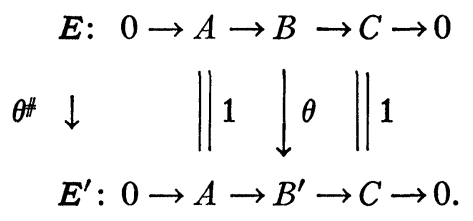

By the 5-lemma, $\theta$ is an isomorphism, and thus $\theta^{*}$ is one too. Thus every endomorphism of $E$ is an "automorphism."

AMS 1970 subject classifications. Primary 13D15, $18 \mathrm{G} 15$.

Key words and phrases. Endomorphism, exact sequence, automorphism, automorphism group, semigroup, isomorphism.

1 This research was partially supported by NSF grant GP-9631. 
The category $\mathfrak{F}$ of all sequences of length two beginning with $A$ and ending at $E$ can be similarly defined. Not all morphisms of $F$ need be isomorphisms however.

Let $\mathfrak{T}^{2}$ denote the abelian category whose objects are all the morphisms of $\mathfrak{M}$, and whose morphisms are all pairs $\left(\begin{array}{c}\rho \\ \sigma\end{array}\right): h \rightarrow g$ which give rise to commutative squares

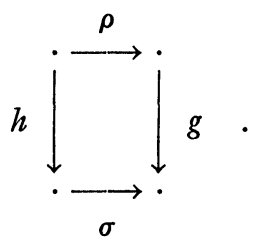

One should note that there is no way of adding endomorphisms in $\mathcal{E}$ and $\mathcal{F}$; one may only compose them with each other.

2. Computation of $\operatorname{Aut}_{\varepsilon}(E)$.

Theorem 1. $\operatorname{Aut}_{\varepsilon}(E) \cong \operatorname{Hom}_{\Re}(C, A)$.

Proof. Let $\alpha^{\sharp}=(1, \alpha, 1): E \rightarrow E$. Set $\alpha-1=\lambda$ or $\alpha=1+\lambda$. Since $\alpha f=f,(1+\lambda) f=f+\lambda f=f$, so $\lambda f=0$. Therefore there is a unique morphism $\mu: C \rightarrow B$ such that $\lambda=\mu g$; so $\alpha=1+\mu g$. But $g \alpha=g$ implies $g(1+\mu g)=g+g \mu g=g$, so $g \mu g=0$. But $g$ is an epimorphism, so $g \mu=0$. Therefore there is a unique morphism $\nu: C \rightarrow A$ such that $\mu=f \nu$. It follows that $\alpha=1+f \nu$. Moreover, $\nu$ is unique because if $\alpha=1+f \nu g$ $=1+f \nu^{\prime \prime} g$ then $f \nu g=f \nu^{\prime \prime} g$; but $f$ is a monomorphism, so $\nu g=\nu^{\prime \prime} g$; similarly $g$ is an epimorphism, so $\nu=\nu^{\prime \prime}$.

This construction produces a unique mapping

$$
\Phi: \operatorname{Aut}_{\varepsilon}(E) \rightarrow \operatorname{Hom}(C, A)
$$

where $\Phi\left(\alpha^{\#}\right)=\nu$. The former is a multiplicative group, the latter an additive abelian group. We must prove that $\Phi$ is an isomorphism of groups.

If $\rho: C \rightarrow A$ is any morphism of $\operatorname{Hom}(C, A)$, then $(1+f \rho g)^{\sharp}=(1$, $1+f \rho g, 1)$ is an automorphism of $E$ because it clearly gives rise to an appropriate commutative diagram. Hence it follows that $\Phi\left((1+f \rho g)^{*}\right)$ $=\rho$, so $\Phi$ is onto.

If $\Phi\left(\alpha^{\sharp}\right)=\Phi\left(\alpha_{1}^{\sharp}\right)=\nu$, then $\alpha=1+f \nu g=\alpha_{1}$, so $\alpha_{1}^{\sharp}=\alpha^{\sharp}$. Therefore $\Phi$ must be a one-to-one mapping.

If $\alpha=1$ then $1^{\#}$ is the identity morphism on $E$ in $\varepsilon$, and $\Phi\left(1^{*}\right)=0$. Certainly $\alpha^{-1}=1-f \nu g$.

Notice that $(1+f \eta g)(1+f \rho g)=1+f \eta g+f \rho g=1+f(\eta+\rho) g$. Therefore, if $\alpha=1+f \eta g$ and $\alpha^{\prime}=1+f \rho g$, then $\Phi\left(\alpha^{\sharp} \alpha^{\prime \prime}\right)=\eta+\rho=\Phi\left(\alpha^{\sharp}\right)$ 
$+\Phi\left(\alpha^{\prime \prime}\right)$. Therefore $\Phi$ is a homomorphism of groups, and in fact an isomorphism.

It is interesting to note that these automorphism groups are abelian, and are independent of the extension class of $E$.

Corollary. $\operatorname{Aut}_{8}(E)$ is isomorphic to a (commutative) subgroup of $\operatorname{Autgr}_{\text {Tr }}(B)$.

There is a subgroup of $\operatorname{Autri}(B)$ of this type for each submodule $A^{\prime}$ of $B$, where the short exact sequence would be $0 \rightarrow A^{\prime} \rightarrow B \rightarrow B / A^{\prime}$ $\rightarrow 0$. If $\operatorname{Autrr}_{(}(B)$ is the direct limit of the $\operatorname{Aut}_{\varepsilon}(E)$ groups, then it would have to be abelian.

In a following paper, Theorem 1 will be used to prove that the Whitehead group of $\mathcal{E}$ is $\operatorname{Hom}(C, A)$.

3. Computation of $\operatorname{End}_{\mathscr{F}}(F)$. By the same argument as was used in Theorem 1 , if $(1, \beta, \gamma, 1): F \rightarrow F$ is an endomorphism of $F$, then there is a unique morphism $\rho: C \rightarrow B$ such that $\beta=1+\rho g$ and a unique morphism $\sigma: D \rightarrow C$ such that $\gamma=1+h \sigma$.

There are two endomorphisms $C \rightarrow C$ produced by this, $g \rho$ and $\sigma h$. One has

$$
\begin{aligned}
h(1+\sigma h) g & =h g+h \sigma h g=(1+h \sigma) h g=\gamma h g \\
& =h g \beta=h g(1+\rho g)=h g+h g \rho g .
\end{aligned}
$$

Therefore $h g \rho g=h \sigma h g$, and since $g$ is an epimorphism and $h$ is a monomorphism, $g \rho=\sigma h$.

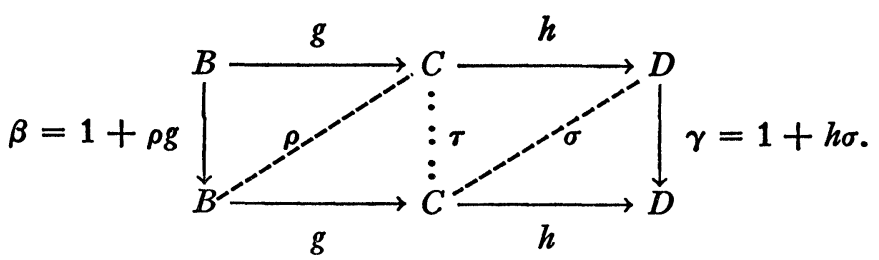

Therefore there is induced a unique morphism $\tau=1+g \rho=1+\sigma h$ : $C \rightarrow C$ such that $\tau g=g \beta$ and $h \tau=\gamma h$. Also, there is a commutative diagram

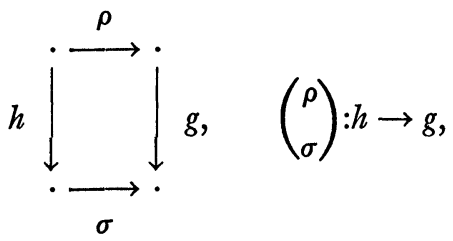

corresponding to a morphism in the category $\mathfrak{T C}^{2}$. 
If we define a multiplication on $\operatorname{Hom}_{\mathfrak{N}^{2}}(h, g)$ by setting

$$
\left(\begin{array}{l}
\rho \\
\sigma
\end{array}\right)\left(\begin{array}{l}
\rho^{\prime} \\
\sigma^{\prime}
\end{array}\right)=\left(\begin{array}{l}
\rho+\rho^{\prime}+\rho g \rho^{\prime} \\
\sigma+\sigma^{\prime}+\sigma h \sigma^{\prime}
\end{array}\right),
$$

then it is easily checked that this is associative and that $\left(\begin{array}{l}0 \\ 0\end{array}\right)$ is a unit with respect to this multiplication. Therefore, using the same techniques as in Theorem 1 , one proves

THEOREM 2. There is an isomorphism of semigroups

$$
\operatorname{End}_{\mathcal{F}}(F) \cong \operatorname{Hom}_{\pi^{2}}(h, g) \text {. }
$$

It would be very interesting if one could give some way of deciding which endomorphisms are automorphisms. An equivalent problem is to determine when $g \rho$ is quasi-regular in $\operatorname{Aut}_{\mathfrak{N}}(C)$.

\section{REFERENCES}

1. S. Mac Lane, Homology, Die Grundlehren der math. Wissenschaften, Band 114, Academic Press, New York; Springer-Verlag, Berlin, 1963. MR 28 \#122.

2. I. S. Pressman, The Baer sum functor and algebraic $K$-theory (to appear).

3. - Whitehead groups of the category of short exact sequences (in prep.).

Ohio State University, Columbus, Ohio 43210 